\title{
Successful Treatment with Surfactant in Preterm Infants with Pulmonary Hemorrhage
}

\author{
ZhihuiRong, Wei Liu, Ling Chen and Liwen Chang* \\ Department of Pediatrics, Huazhong University of Science and Technology, China
}

*Corresponding author: Liwen Chang, Department of Pediatrics, Tongji Hospital, Tongji Medical College, Huazhong University of Science and Technology, Wuhan, China

\begin{abstract}
Pulmonary hemorrhage (PH) is a life threatening condition in newborns, especially preterm infants. Surfactant replacement might be helpful to improve lung function and reduce the ventilation support in infants with PH. We report two premature infants (one with early onset PH and one with late onset PH) who have been successfully rescued by administering surfactant (Calsurf) after an acute episode of bleeding. Both of the 2 infants survived, and surfactant administration showed improvement in mean airway pressure and oxygenation indices. In the current study Calsurf was found effective in the treatment of both early PH and late $\mathrm{PH}$ in premature infants. There were no potential side effects were found in our study.
\end{abstract}

Keywords: Premature infants; Pulmonary hemorrhage; Surfactant

\section{Introduction}

Pulmonary hemorrhage $(\mathrm{PH})$ is a life threatening condition occurring to 1-12/1000 live births, with rates increasing to 50/1000 live births in risk groups, such as prematurity, intrauterinegrowth restriction (IUGR), patent ductus arterosus (PDA) and/or sepsis [1]. Small gestational age, low birth weight, low agar score at 1 and $5 \mathrm{~min}$, severe respiratory distress syndrome (grade 3 or 4 ) and use of surfactant replacement are risk factors of PH [2]. Currently the treatment of $\mathrm{PH}$ includes stopping pulmonary hemorrhage by epinephrine spraying through an ET, treating secondary RDS by optimal ventilation settings with or without surfactant [3]. On one hand, pulmonary hemorrhage is thought to be a complication of surfactant therapy because surfactant can rapidly lower the intrapulmonary pressure, which facilitates left to right shunting through PDA and an increase in pulmonary blood flow. Thus, clinicians might hesitate to administer surfactant again in neonates with $\mathrm{PH}$. While on the other hand, the presence of hemoglobin, red cell membrane lipid and serum proteins can increase surface tension resulting from surfactant dysfunction and cause secondary RDS [4]. Surfactant replacement might be helpful to improve lung function and reduce the ventilation support. Calsurf has been used in China for several years [5]. Calsurf is a newborn bovine lung surfactant extract as a lyophilized powder in a $70 \mathrm{mg} /$ vial. The clinical trials in China by Kong et al. found that the prophylactic use of a domestic surfactant preparation is better than early surfactant treatment in improving the pulmonary status and decreasing the incidence of severe RDS and duration of mechanical ventilation [6].

\section{Case Report}

\section{Case1: Early onset of PH}

A 30-week male neonate with birth weight of 1670 grams was admitted in neonatal intensive care unit. He showed respiratory distress shortly after birth and was diagnosed of grade 4 respiratory distress syndrome (RDS) by chest X ray. Three doses of surfactant (Calsurf, Shuanghe Famaceutics, China, 80mg/kg.d)were given and he was successfully extubated to SiPAP on day 3. On day 5 of age, the neonate developed profuse $\mathrm{PH}$. He showed desaturation $\left(\mathrm{SpO}_{2} 60 \%\right)$ and when intubated, a large amount of fresh blood was noticed in the endotracheal tube. Chest X ray showed PH (Figure 1). Coagulation tests were normal. Echo showed no PDA. The neonate was put on conventional ventilator with high setting (SIMV, 25/7, $\mathrm{FiO}_{2} 1.0$ ) and $\mathrm{SpO}_{2}$ fluctuating from 80-90\% (Oxygen indice was 40, see Table 1). Two doses of Calsurf $(40 \mathrm{mg} / \mathrm{kg}$ with dosing interval of $12 \mathrm{hr}$ ) were given. Clinical and radiological improvement (Figure 2) were found after $24 \mathrm{hr}$ of surfactant treatment (Oxygen indice 
was 170). The neonate was successfully extubated on day 7 . At the meanwhile, antibiotics, total fluid limitation were also given to this patient. This infant discharged home on day 30 (CGA 34W) without
BPD, PVL and ROP. Flow diagram of the patient till discharge was shown on (Figure 3).

Table 1: Blood and ventilator setting before and 1h of first PS was given.

\begin{tabular}{|c|c|c|c|c|c|}
\hline & Parameter s & Patient 1 Before PS & Patient 1 1h after PS & Patient 2 Before PS & Patient 2 1h after PS \\
\hline ABG & PH & 7.1 & 7.35 & 6.9 & 7.28 \\
\hline & CO2 & 65 & 45 & 40 & 55 \\
\hline & O2 & 39 & 68 & -15 & -8 \\
\hline Ventilator & BE & -12 & SIMV & HFV & HFV \\
\hline & MODE & SIMV & $20 / 6$ & 15 & 12 \\
\hline
\end{tabular}

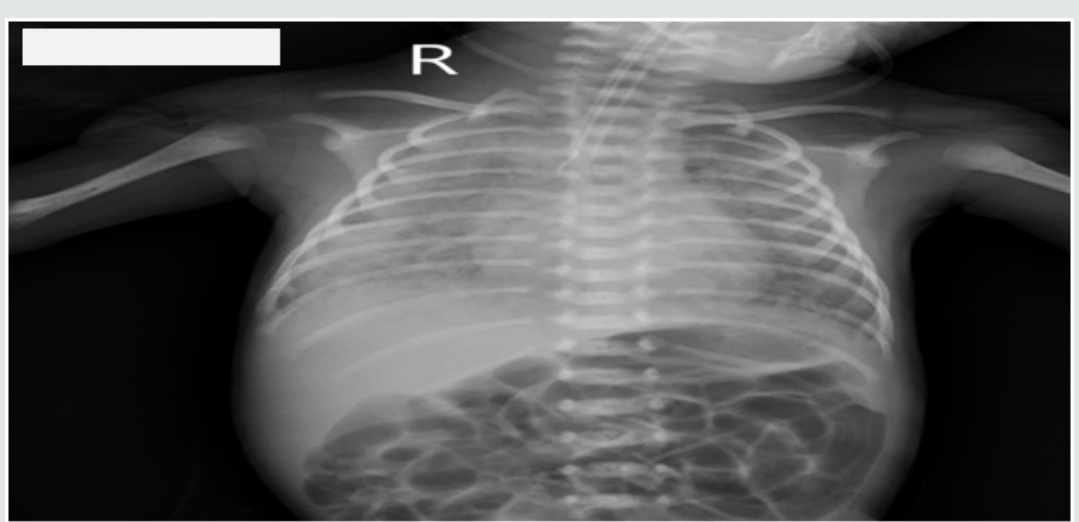

Figure 1: Case 1 pre surfactant chest $X$ ray.

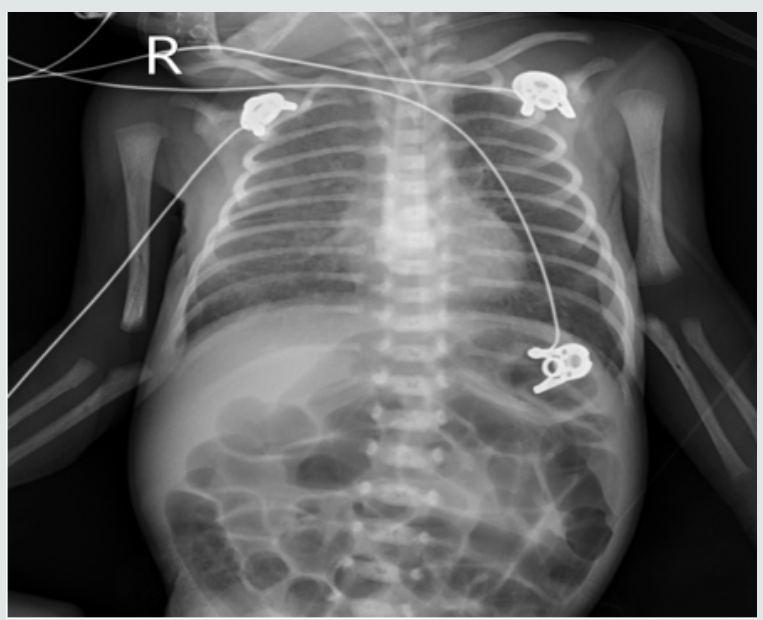

Figure 2: Case 1 post surfactant chest $X$ ray.

\section{Case 2: Late onset of PH}

A 29-week neonate born to a mother with nephritic syndrome was admitted in neonatal intensive care unit. His birth weight was 1100 grams. He showed respiratory distress shortly after birth and diagnosed of grade 2 RDS. INSURE (Intubate-surfactantextubate to SiPAP) technique was used. Patent ductus arteriosus, hyperbilirubinemia, Apnea and sepsis complicated with initial course. On day $17^{\text {th }}$ of life, the neonate developed $\mathrm{PH}$ and desaturation (Sp0250-60\%). He was intubated immediately, and a large amount of fresh blood was noticed in the endotracheal tube. Chest $\mathrm{X}$ ray showed PH (Figure 3). The ventilator settings on him were HFV, MAP15, $\mathrm{FiO}_{2} 1.0$ to get the saturation around 90\% (Table 1). Lab results showed that this neonate had severe infection. Two doses of Cal surf ( $60 \mathrm{mg} / \mathrm{kg}$ with dosing interval of $12 \mathrm{hr}$ ) were given. Besides ventilation and PS, antibiotics and RBC blood transfusion were also given to this neonate. 
Figure 3 Flow chart of patient 1 till discharge

\section{GA30W, BWT1670g}

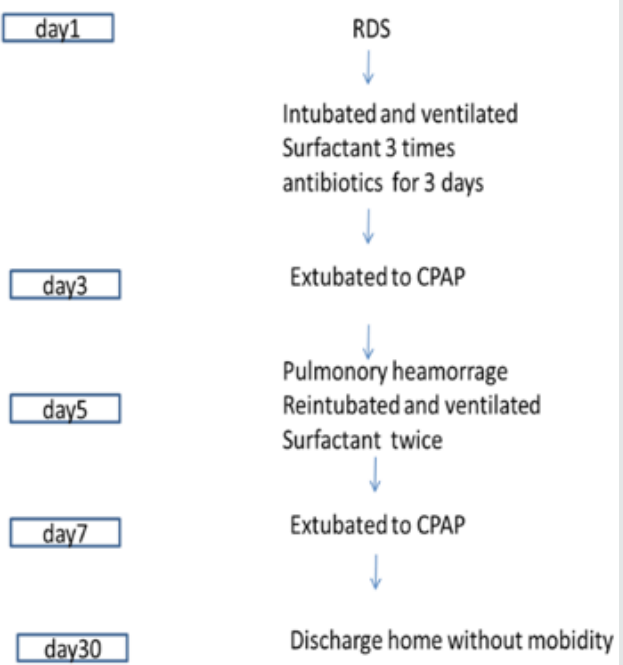

Figure 3: Flow diagram of the patient 1 till discharge.

Clinical and radiological improvement (Figure 4) were found after $24 \mathrm{hr}$ of surfactant treatment. The neonate was successfully

extubated on day 21. This infant discharged home on day 64 (CGA $38 \mathrm{~W}$ ) with moderate BPD, but without PVL and ROP.

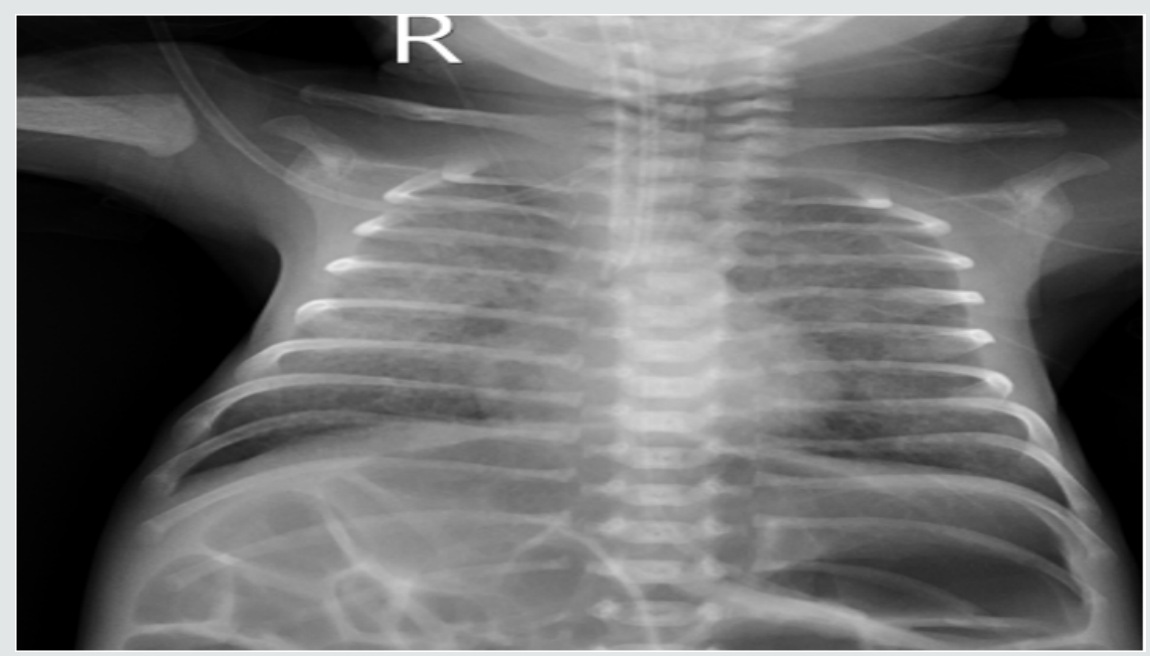

Figure 4: Case 2 pre surfactant chest $X$ ray.

\section{Discussion}

Pulmonary hemorrhage is a life-threatening condition and more prevalent in premature newborns, and is associated with mechanical ventilation, PDA, infection and previous use of blood components [2]. Both of our two cases are premature newborns, one suffered from PH shortly after birth ( $5^{\text {th }}$ day of life), the other one suffered from $\mathrm{PH}$ on day $17^{\text {th }}$ of life, most probably due to severe nosocomial infection (Figure $5 \& 6$ ). Different approaches have been proposed for the management of $\mathrm{PH}$, including stopping pulmonary hemorrhage by epinephrine spraying through an ET, treating secondary RDS by optimal ventilation settings with or without surfactant [3]. Invitro study showed that the presence of surfactant impaired coagulation by decreasing platelet aggregation and increasing prothrombin time (PT). Whole blood thromboelastogram studies also showed a trend towards lengthening of clotting time with increased surfactant concentration and lower clot strength [5]. Thus, clinicians might hesitate to administer surfactant again in neonates with PH. PH has been effectively managed by using surfactant instillation. Senol et al. reported two kinds of natural surfactants improved oxygenation when administered for PH in very low birth weight infants [6]. In majority of cases studies surfactant was given as a treatment of $\mathrm{PH}$ in the first few days of life (early onset of PH) [6,7]. However, in the present study both early onset of $\mathrm{PH}$ and late onset of $\mathrm{PH}$ were successfully rescued by surfactant therapy. In our two cases, Calsurf was used in both cases and Calsurf administration showed improvement in mean airway pressure and oxygenation indices. 
Calsurf is a natural surfactant produced by Shuang he Famaceutics, China. Its active ingredient is the phospholipid fraction obtained from bovine lung. Of the surfactant lipid, total phospholipid is more than $80 \%$, of which phosphatidylcholine is no less than $55 \%$. Calsurf has been found effective in the treatment of respiratory distress syndrome among premature infants in Chinese population $[8,9]$. In the current study Calsurf was found effective in the treatment of $\mathrm{PH}$ in premature infants.

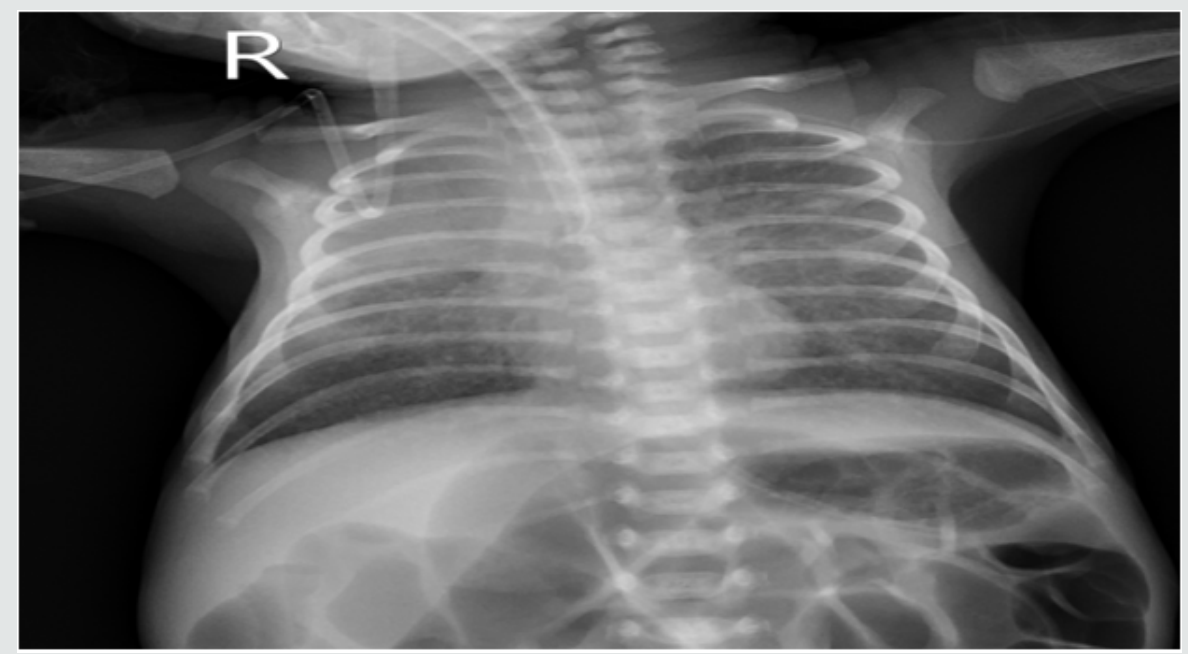

Figure 5: Case 2 post surfactant chest $X$ ray.

\begin{tabular}{|l|}
\hline Figure 6 Flow chart of patient 2 till discharge \\
\hline GA29W, BWT1100g \\
\hline day1 \\
day3 \\
antibiotics \\
PDA successfully treated \\
with Ibuprofen \\
day \\
day21 \\
Pulmonory heamorrage \\
Reintubated and ventilated \\
Surfactant twice \\
dav64 \\
Extubated to CPAP
\end{tabular}

Figure 6: Flow diagram of the patient 2 till discharge.

\section{Conclusion}

Surfactant (Calsurf) therapy was found to be safe and effective in both early PH and late PH in preterm infants. Further randomized controlled trail would be useful for identification of potential benefit.

\section{References}

1. Suryawanshi P, Nagpal R, Meshram V, Malshe N, Kalrao V (2015) Pulmonary Hemorrhage (PH) in Extremely Low Birth Weight (ELBW) Infants: Successful Treatment with Surfactant. J Clin Diagn 9(3): 3-4.

2. Ferreira CH, Carmona F, Martinez FE (2014) Prevalence risk factors and outcomes associated with pulmonary hemorrhage in newborns. J Pediatr (Rio J) 90(3): 316-322.
3. Yen TA, Wang CC, Hsieh WS, Chou HC, Chen CY, et al. (2013) Shortterm outcome of pulmonary hemorrhage in very-low-birth-weight preterm infants. Pediatr Neonatol 54(5): 330-334.

4. Aziz A,

OhlssonA

(2012)

Surfactant for pulmonary haemorrhage in neonates. Cochrane Database Syst.

5. Kong X, Cui Q, Hu Y, Huang W, Ju, et al. (2016) Surfactant Replacement Therapy in Neonates of Less than 32 Weeks' Gestation: A Multicenter Controlled Trial of Prophylaxis versus Early Treatment in Chinaa Pilot Study. Pediatr Neonatol 57(1): 19-26.

6. Sankar MJ, Gupta N, Jain K, Agarwal R, Paul VK (2016) Efficacy and safety of surfactant replacement therapy for preterm neonates with respiratory distress syndrome in low- and middle-income countries: a systematic review. J Perinatol 36(1): S36-S48. 
7. Strauss T, Rozenzweig N, Rosenberg N, Shenkman B, Livnat T, et al. (2013) Surfactant impairs coagulation in-vitro: a risk factor for pulmonary hemorrhage? Thromb 132(5): 599-603.

8. Bozdağ Ș, Dilli D, Gökmen T, Dilmen U (2015) Comparison of two natural surfactants for pulmonary hemorrhage in very low-birth-weight infants: a randomized controlled trial. Am J Perinatol 32(3): 211-218.
9. Amizuka T, Shimizu H, Niida Y, Ogawa Y (2003) Surfactant therapy in neonates with respiratory failure due to haemorrhagic pulmonary oedema. Eur J Pediatr 162(10): 697-702.

\section{(c) \\ This work is licensed under Creative Commons Attribution 4.0 License}

To Submit Your Article Click Here:

Submit Article

DOI: $10.32474 /$ PAPN.2019.02.000137

$\begin{gathered}\text { Progressing Aspects in Pediatrics } \\ \text { and Neonatology } \\ \text { Assets of Publishing with us } \\ \text { Global archiving of articles }\end{gathered}$
- Immediate, unrestricted online access
- Rigorous Peer Review Process
- Authors Retain Copyrights

